\title{
Smart Ambulance Approach Alarm System Using Smartphone
}

\author{
Toru KOBAYASHI ${ }^{\dagger a}$, Fukuyoshi KIMURA ${ }^{\dagger}$, Tetsuo IMAI ${ }^{\dagger}$, and Kenichi ARAI ${ }^{\dagger}$, Members $^{2}$
}

\begin{abstract}
SUMMARY In order to operate an ambulance efficiently, we developed a Smart Ambulance Approach Alarm System using smartphone, by notifying the approach of an ambulance to other vehicles on public roads. The position information of ambulances has not been opened in view of development costs and privacy protection. Therefore, our study opens the position information inexpensively by loading commodity smartphones, not special devices, into ambulances. The position information is made to be open as minimum necessary information by our developed cloud server application, considering dynamic state of other vehicles on public roads and privacy of ambulance service users. We tested the functional efficiency of this system by the demonstration experiment on public roads.

key words: IoT, ambulance, information of position, open data
\end{abstract}

\section{Introduction}

According to a survey by Ministry of Internal Affairs and Communications, there were $6,054,815$ ambulance responses during 2015, and the number of people taken to hospitals by ambulances reached 5,478,370 [1]. Because of its limited area, concentration of the Japanese population in urban area causes frequent traffic jam [2]. Moreover, the high temperature and high humidity during recent summer in Japan make drivers close its windows and turn on the air conditioner. Drivers must keep the law that they are to recognize approaching ambulances even in summer period. However, road conditions change every second. For example, we also might have noisy neighbor vehicles such as big trucks or noisy construction sites along the road. As a result, the situation occurs that drivers are unable to hear the siren of an ambulance. This causes delays in the availability of ambulance for emergency.

In order to solve this problem, we developed Smart Ambulance Approach Alarm System using smartphone. The information of ambulance positions has not been opened in view of development cost and privacy protection yet. In terms of development cost, the position information of ambulances is inexpensively opened, because it is realized just by loading a commodity smartphone and we do not have to develop special embedded devices nor customize an original ambulance to adapt special embedded devices. It concludes our proposed system makes ambulances IoT just by installing smartphones with our developed application.

\footnotetext{
Manuscript received November 9, 2018.

Manuscript revised March 25, 2019.

Manuscript publicized June 21, 2019.

${ }^{\dagger}$ The authors are with Graduate School of Engineering, Nagasaki University, Nagasaki-shi, 852-8521 Japan.

a)E-mail: toru@ cis.nagasaki-u.ac.jp

DOI: $10.1587 /$ transinf.2018OFL0003
}

In terms of privacy issues, the ambulance position information is sent to general vehicles that are to be informed the approach of the ambulance while the ambulance runs on main road such as national road. When the ambulance runs into community road from main road, the ambulance position information is not opened any more.

We assume that the smartphone used in this system is located in a glovebox mounted on an ambulance. In this sense, the smartphone is positioned the same as embedded devices because ambulance crew has to do nothing.

\section{Related Works}

We have some studies on leading an ambulance into a hospital smoothly. P. Attri proposed the system that preempts signals in the direction in which an ambulance is heading [3]. In this system, preempting signals by mobile devices prevents access delay to hospitals. E. Mouhcine suggested the system that shows the best-suited route to hospitals [4]. This system features algorithm that calculates the most suitable routes using congestion situations, the speed limit, and the number of available ambulances and location information of hospitals. However, these systems do not aim to open the position information of ambulances to other vehicles, focuses on improving efficiency of ambulances by calculating the most suitable route or preempting the signals.

We have the practical use system to streamline operation of emergency vehicle, utilizing the position information of fire engines and ambulances. In Attica, Greece, the system which dispatches effectively ambulances is put in operational service by coordinating GIS (Geographic Information System), GPS (Global Positioning System) and GSM (Global System for Mobile Communication) [5]. However, this existing system needs special devices to be installed on emergency vehicles. In addition, the position information of emergency vehicles is shared among only Firefighting Command Center, not opened to the public. These are different points from our aims.

On the other hand, there is the study that aims to detect the sirens of emergency vehicles (ambulance, fire engine and patrol car) in the outdoor environment and to inform drivers of its presence [6]. However, the volume of the siren depends on situation such as neighboring noise and vehicle window opening and shutting state. Therefore, we have a difficulty to manage it stably. 


\section{Smart Ambulance Approach Alarm System}

\subsection{Requirements and Double Phase Filtering Model}

To realize our system, we need to specify requirements from the aspects of operating ambulances, user of ambulance services, and general vehicle drivers who should recognize the approach of an ambulance.

- Requirement 1: capable of transmitting the position information of ambulances only during the emergency situation, not remodeling an ambulance itself, nor tasking firefighters.

- Requirement 2: capable of respecting privacy not as specifying the ambulance service users.

- Requirement 3: capable of informing the position information of ambulances only to the general vehicles which should recognize the approach of the ambulance.

Figure 1 shows the double phase filtering model that satisfies these requirements. The first phase filter extracts ambulances on emergency, and that position information is put into the second phase filter. Consequently, the only general vehicle that should be notified the approach of the ambulance is filtered. It is a red vehicle in Fig. 1.

\subsection{Basic System Configuration}

This system consists of smartphones on ambulances and general vehicles, and a cloud server that links these smartphones (Fig. 2). These smartphones and a cloud server are each installed our developed applications, for ambulances, general vehicles and a cloud server. The ambulance smartphone application has the function that sends the position information of ambulances to the cloud server only during the emergency situation (1) Fig. 2). Whether the ambulance is in the state of emergency or not is judged on sensing the sirens of ambulances by the ambulance smartphone application. This makes it possible to transmit the position information of ambulances to the cloud server, just by putting a smartphone on the dashboard of ambulance with the application active. This has the merits of cost: it does not require adding special interface devices on an ambulance, and operation: it does not require firefighter's time and efforts (Requirement 1)

On the other hand, the cloud server application notifies the position information of ambulances to other general vehicles only when the following conditions are all satisfied.

- Condition 1: an ambulance goes on main roads.

- Condition 2: the distance between the ambulance and other general vehicles is less than 500 meters.

- Condition 3: general vehicles and the ambulance are close together.

Condition 1 prevents it from opening the position information even if the ambulance arrives at a house on community

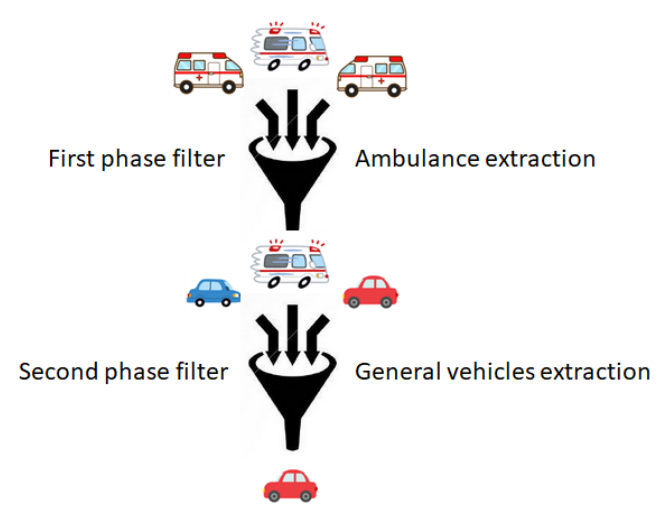

Fig. 1 Double phase filtering model.

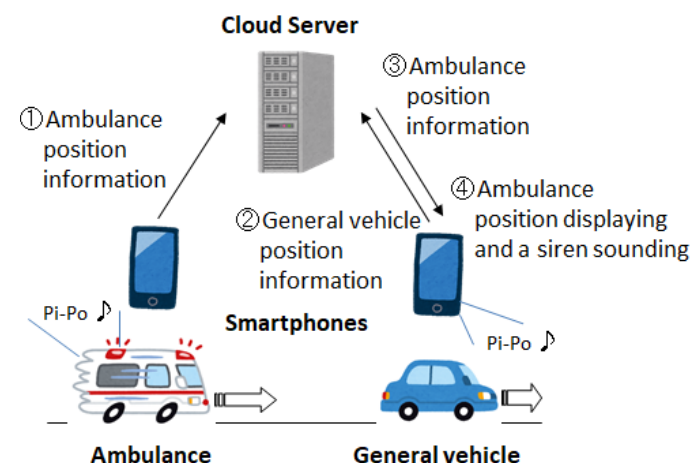

Fig. 2 Smart ambulance approach alarm system.

Table 1 System specifications.

\begin{tabular}{|l|l|l|l|}
\hline & \multicolumn{1}{|c|}{ Ambulance } & General Vehicle & \multicolumn{1}{c|}{ Cloud Server } \\
\hline $\begin{array}{l}\text { Hard- } \\
\text { ware }\end{array}$ & $\begin{array}{l}\text {-Sharp AQUOS EVER } \\
\text { SH-02J }\end{array}$ & $\begin{array}{l}\text {-Sharp AQUOS } \\
\text { EVER SH-02J }\end{array}$ & $\begin{array}{l}\text {-CPU: Intel(R) Xeon(R) CPU E3- } \\
1220 \text { v3 @ 3.10GHz cpu cores: } 4 \\
- \text { Memory: 8GB }\end{array}$ \\
\hline $\begin{array}{l}\text { Soft- } \\
\text { ware }\end{array}$ & $\begin{array}{l}\text {-Android 7.0 } \\
\text {-JAVA 1.8.0 (JDK8) } \\
\text {-Android Studio 3.0.1 }\end{array}$ & $\begin{array}{l}\text {-Android 7.0 } \\
\text {-Google } \\
\text { Chrome }\end{array}$ & $\begin{array}{l}\text { - Cent OS 7.3 / Apache 2.4 } \\
\text { - PostgreSQL 9.5 / PHP5.6 }\end{array}$ \\
\hline
\end{tabular}

roads (Requirement 2). Owing to condition 2 and 3, the position information of ambulances is sent only to general vehicles that are required to clear the way for the approaching ambulance (Requirement 3).

To decide whether these conditions are satisfied or not, the general vehicle application transmits its own position information to the cloud server application (2) Fig. 2). If the above mentioned conditions are all satisfied, the position information of ambulances is transmitted to the general vehicle smartphone applications (3) Fig. 2). The position information of ambulances is displayed on the maps by the general vehicle smartphone applications as the simulated sirens sound.

\section{Prototype System}

Table 1 shows the hardware and software specifications for prototype system. 


\subsection{Ambulance Smartphone Application}

This application collects the sound from microphone of smartphone and judges whether the ambulance sounds a siren or not by frequency analyzing the collected sound through Fourier transform. For the recording, this application uses the raw mode, and the data are stored in little endian. However, the program through Fourier transform is supposed to the big endian for the order of data storage, malfunction will occur when Fourier transform is applied as it. In order to prevent this malfunction, Fourier transform is applied after endian transform. Real Discrete Fourier Transform from Ooura FFT library in C programming language is implemented in Java and the Android application is in Java as well [7].

\subsection{General Vehicles Smartphone Application}

This is implemented as web application. This acquires the latitude and longitude information about the present position through the GPS functions built in smartphones, and transfers to the cloud server application. In addition, it displays its own position on google map. When the position information of ambulances is gained from the server, the position of ambulances is shown on the same map and it makes the simulated siren sound. This process is implemented every 2 seconds. The simulated siren sound enables general vehicle drivers to know the approaching ambulances without seeing the smartphone. This will contribute to safety driving in the same way as a car navigation system.

\subsection{Cloud Server Application}

Based on the requests from the general vehicle smartphone application, the position information of ambulances is transmitted to the general vehicle smartphone application only when all the conditions shown in chapter 3.2 are satisfied. When the ambulance goes away from the own vehicle or is far with it more than 500 meters, the position information of ambulances is not reported (Fig. 3). Therefore, the icons actually displayed on the general vehicle smartphone application as the simulated siren sounds, are only red ones in Fig. 3.

\section{Evaluation Experiment}

We experimented this prototype system on public roads around Bunkyo Campus, Nagasaki University on 14th May, 2018, using 2 general vehicles. One vehicle plays a role as an ambulance, that is mounted with the ambulance smartphone application installed smartphone and the laptop that sounds the simulated sirens (Fig. 4). The other vehicle acts as a general vehicle that is loaded with the smartphone the general smartphone application installed. Figure 5 shows the experiment environment. This experiment was done under the state that the vehicle with role of the general vehicle

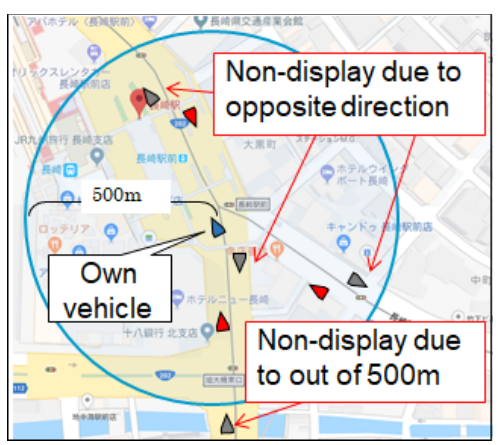

Fig. 3 Ambulance position display conditions.

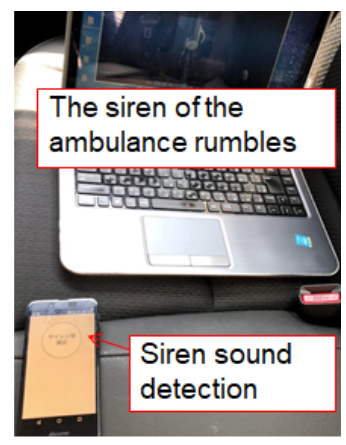

Fig. 4 Ambulance simulation equipment.

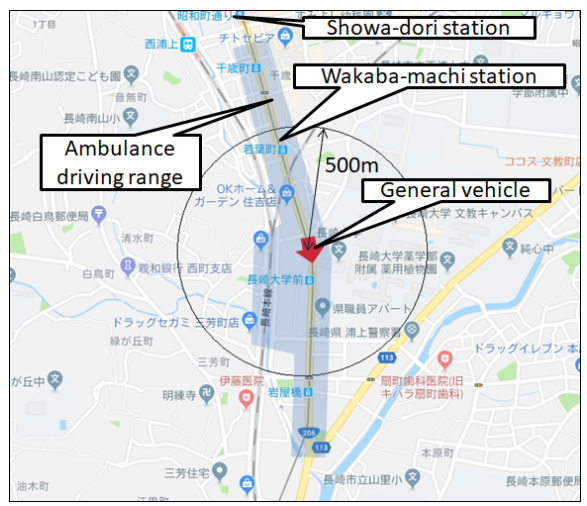

Fig.5 Experiment environment.

was parked in front of the main gate of Bunkyo Campus, toward south. Regarding the roads along the City tramline, indicated in yellow, stretching north and south on the map as the main roads, the vehicle with role of the ambulance went on these roads and community roads around Campus (blue shaded area, Fig. 5).

When the ambulance smartphone application detected the simulated sirens which on-board laptop sounded, the screen shown in Fig. 4 was displayed on the smartphone. Thereby we confirmed that the position information of the ambulance was sent to the cloud server. In case the vehicle of the ambulance role went southward from Showa-dori station along the tramline, it was confirmed that smartphone of the general vehicle role started to display the position of the ambulance as passing through Wakaba-machi station (Fig. 6). Furthermore, the information of the ambulance was not shown on the screen in the south of the point where the ambulance role vehicle overtook the vehicle of the general vehicle role: in front of the main gate of Bunkyo Campus. Additionally, in case that the ambulance role vehicle went on the community roads, it was proved that the position of the ambulance was not displayed on the smartphone of the vehicle with general vehicle role, even the distance between the ambulance and the general vehicles was less than 500 meters.

In terms of usability for general vehicle drivers, we conducted interviews after showing them four methods to 


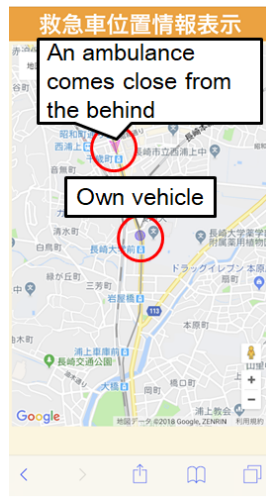

Fig. 6 Display image

notify approaching ambulances.

1. Loud siren sound from beginning to end

2. Initially low sound, then gently loud sound

3. Voice message, such as "Please be careful, an ambulance is approaching."

4. Combination of method 2 and 3.

We asked 10 people, men and women in their 20's to 50's, who have driver licenses. The question is "which method is the most suitable to notify approaching ambulances?". $60 \%$ of them answered that method 4 was the most suitable and method 3 was chosen by $40 \%$. Although method 1 is adopted in our prototype system, it was not chosen for the suitable way. The reason why method 1 was not selected is that the unexpected loud sound surprises drivers.

\section{Discussion}

Concerning requirement 1 , once the ambulance smartphone application is activated, it is possible to notify automatically the position information of ambulances by whether the siren of the ambulance is sounded or not. This satisfies the requirement 1 .

On evaluating requirement 2 , we drove the vehicles on both the road along the tramline and community roads, and confirmed that the ambulance smart application transmitted the position information of the ambulance only when it went on the tramline. Therefore, this means this system considers privacy so as not to specify the ambulance service users. However, it does not protect the privacy of users who live along the main roads; this means it does not completely satisfy Requirement 2 . To protect privacy definitely, we need to establish an operation rule that the ambulance crew stops the siren when the ambulance approaches the patient's house along the main road. If the siren automatically stops, it is better solution.

On regarding Requirement 3, the position information of ambulances was sent only to the vehicles which were considered necessary to give way to the ambulance, which was about to get close as shown in Fig. 5. For this reason, we confirmed that this system satisfied Requirement 3. However, the position information of ambulances was transmitted to the general vehicles only if the vehicle used our developed general vehicle smartphone application. For this reason, it is required to be applicable to the car navigation devices or car navigation applications for the smartphones.

As an additional evaluation, this system is able to gather information about traveling routes of an emergency vehicle, the number of nearby general vehicles or a traffic jam as big data. Using this big data, we can analyze traveling routes which are frequently congested. This information helps an ambulance operation to choose a traveling route avoiding a heavy traffic jam.

\section{Conclusion}

We developed the Smart Ambulance Approach Alarm System. This is characterized by low cost and easy as it opens the position information of ambulances on emergency using smartphones. By opening the position information of ambulances within a necessary minimum range, this system becomes possible to be privacy conscious of the ambulance service users. The evaluation experiment on public roads using prototype verifies the validity of these characteristics. For further study, we improve the method of notifying approaching ambulances according to the result of user interview as described in Chapter 5. Furthermore, we consider the improvement of the system to protect more privacy of users, and applicability to the car navigation devices and car navigation applications for the smartphones for practical use. Then, we try to have real field experiment in an actual environment using the real ambulances.

\section{References}

[1] Fire and Disaster Management Agency, http://www.fdma.go.jp/ neuter/topics/fieldList9_3_2016.html

[2] The Metropolitan Police Department, http://www.keishicho.metro. tokyo.jp/about_mpd/jokyo_tokei/tokei_jokyo/ippan.html

[3] P. Attri, F. Rafiqui, and N. Rawal, "Traffic Signal Preemption (TSP) system for ordinary vehicles in case of emergency based on Internet of Things ecosystem," 2016 3rd International Conference on Computing for Sustainable Global Development (INDIACom), pp.16-18, 2016.

[4] E. Mouhcine, Y. Karouani, K. Mansouri, and Y. Mohamed, "Toward a distributed strategy for emergency ambulance routing problem," 2018 4th International Conference on Optimization and Applications (ICOA), pp.1-4, 2018.

[5] G. Derekenaris, J. Garofalakis, C. Makris, J. Prentzas, S. Sioutas, and A. Tsakalidis, "An information system for the effective management of ambulances," Proc. 13th IEEE Symposium on Computer-Based Medical Systems. CBMS 2000, pp.269-273, 2000.

[6] I. Dabran, O. Elmakias, R. Shmelkin, and Y. Zusman, "An intelligent sound alam recognition system for smart cars and smart homes," 2018 IEEE/IFIP Network Operations and Management Symposium, pp.1-4, 2018.

[7] Ooura FFT library, http://www.kurims.kyoto-u.ac.jp/\%7Eooura/fft-j. html 